\title{
PERÚ EN EL NIVEL DE GRADO DE INVERSIÓN
}

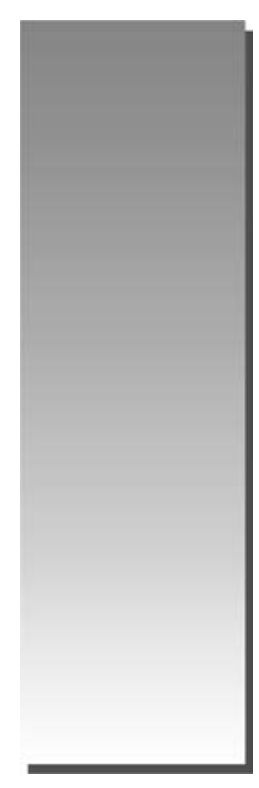

Jorge V. Mayurí Barrón (*)

E-mail: jvmayuri@gmail.com

\section{RESUMEN}

La agencia calificadora de riesgo crediticio Fitch Ratings otorgó al Perú el miércoles 02 de abril del 2008 el primer escalón en el grupo de calificaciones correspondientes al denominado grado de inversión, al elevar la calificación de la deuda peruana de largo plazo en moneda extranjera de "BB+" (grado especulativo) a "BBB-" con perspectiva positiva, Asimismo, elevó la calificación de la deuda en moneda local de largo plazo de "BBB-" a "BBB" también con perspectiva estable, y que ya se encontraba bajo la calificación de grado de inversión.

Este atributo ¿qué beneficios tangibles traerá para la economía del país? ¿mejorará el ingreso per cápita de los peruanos? ¿impulsará la creación de nuevos puestos de trabajo? ¿reducirá el nivel de pobreza del país? ¿por qué sólo México, Chile y ahora Perú son los únicos países que han alcanzado esta categoría? En las líneas que siguen procuraremos dar respuesta a todas y cada una de las interrogantes planteadas.

Palabras clave: Costo de Financiamiento, Deuda Pública, Grado de Inversión, Riesgo Crediticio, Tasas de Interés.

\section{ABSTRACT}

The credit rating agency Fitch Ratings gave Peru on Wednesday, April 2, 2008, the first step in the group ratings for the so-called investment grade, by raising the qualification of the Peruvian longterm debt in foreign currency "BB +" (speculative grade) to "BBB-" with positive outlook, also raised the rating of the debt in local currency long-term "BBB-" to "BBB", also with a stable outlook, and that was already under the classification of investment grade.

This attribute what will bring tangible benefits for the country's economy? ¿can improve the per capita income of peruvians? ¿do encourage the creation of new jobs? ¿can reduce the level of poverty in the country? ¿why just Mexico, Chile and Peru are now the only countries that have reached this category? In the next lines, we will try to respond to each and every one of the questions raised.

Key words: Cost of Financing, Debt, Investment Grade, Credit Risk, Interest Rates.

(*) Doctor en Educación (UNMSM), Magíster en Administración con Mención en Gestión Empresarial (UNMSM) y Licenciado en Administración de Empresas (Universidad Ricardo Palma). Profesor Asociado de la Facultad de Ciencias Administrativas de la UNMSM. 


\section{LA NOTICIA POSITIVA DEL DÍA}

Como sabemos, el grado de inversión o investment grade es asignado a aquellos países que presentan una elevada probabilidad de pago de sus compromisos financieros, lo que les permite acceder a un menor costo crediticio a nivel internacional, y ello se refleja en menores tasas de interés en el país ${ }^{1}$.

Fitch explicó que esta decisión reconoce la fuerte mejora de los indicadores de solvencia externa y fiscal del Perú, que ahora contrarrestan cualquier posibilidad de incumplimiento de pago de sus obligaciones en moneda extranjera. Igualmente, contrarrestan la concentración de las exportaciones peruanas en pocos productos, así como los riesgos políticos y sociales del país.

"Las finanzas públicas de Perú y el desempeño de sus cuentas externas nuevamente superaron las expectativas, contribuyendo a una mejoría mayor que la esperada en sus indicadores financieros en moneda extranjera", afirmó la directora principal de Calificación Soberana para América Latina de Fitch Ratings, Theresa Paiz. Agregó que como resultado de este desempeño, la mayoría de los indicadores financieros de Perú ahora son más fuertes que el promedio de países cuya deuda externa está calificada en los niveles más bajos de grado de inversión.

Más aún, destacó la existencia de un cambio estructural incipiente en los factores que impulsan el crecimiento económico de nuestro país, como el mayor dinamismo de las tasas de crecimiento de los sectores no primarios.

Ya el 19 de octubre del 2007 la agencia calificadora de riesgo crediticio Dominion Bond Rating Service (DBRS) de Canadá otorgó al Perú el grado de inversión (BBB) para su deuda a largo plazo en moneda extranjera y local, por encima de las calificaciones otorgadas al país por las principales agencias, como las estadounidenses.

Fitch es una las principales calificadoras de riesgo del mundo, junto con Standard \& Poor's y Moody's. Standard and Poor's tiene asignada una calificación de BB + con perspectiva estable y dista un peldaño del grado de inversión, mientras que la actual calificación crediticia de Perú asignada por Moody's está un poco rezagada respecto a las otras agencias calificadoras y se sitúa en $\mathrm{Ba} 2$ con una perspectiva estable, y dista dos peldaños del grado de inversión.

\section{QUÉ ES EL GRADO DE INVERSIÓN}

La calificación de la deuda soberana, en este caso BBB-, se refiere a la capacidad de un gobierno de honrar sus deudas con el sector privado. El nivel de rating que Fitch ha asignado al Perú se entiende como un moderado riesgo de crédito o adecuada capacidad de pago. Una mejora en las calificaciones crediticias de Perú reduce el costo de financiamiento, tanto para el sector privado como el público y amplía la gama de potenciales inversionistas dispuestos a realizar operaciones.

Un emisor de deuda se encuentra dentro del grado de inversión cuando los títulos que coloca en el mercado no representan un riesgo de incumplimiento en sus obligaciones para con los compradores de los mismos. Para establecer este grado y estimar la capacidad de pago del país ante posibles shocks y escenarios internacionales negativos, se miden los ingresos futuros, la estabilidad fiscal y la estabilidad macroeconómica y política.

Conseguir esta calificación es importante y beneficiosa para un país por varias razones: primero, porque ayuda a crear un mejor ambiente de negocios pues genera credibilidad en los inversionistas extranjeros sobre el futuro de sus inversiones dentro del país. A su vez, les permite establecer inversiones de más largo plazo, debido a que pueden establecer previsiones de una manera más acertada. Por otro lado, permite que el país obtenga financiamiento en el extranjero en mejores condiciones, dada la reducción de la prima de riesgo que los países en desarrollo deben pagar por su mayor probabilidad de impago y mal historial crediticio, y entre mejor calificado un país, una empresa o una entidad pública, mayor acceso tendrá a créditos con menores tasas de interés. Hay más confianza en su capacidad de pago, ergo, es menor el pago por el riesgo. Las calificaciones van desde la " $D$ " (las peores) hasta una triple "A"; estar en "A" significa ser un país prestigiado, digno de préstamos y de inversión. De esta manera, el Perú se suma a un pequeño grupo de países de la región como Chile y México que tienen grado de inversión, a los que recientemente se ha incorporado Brasil.

1 Ver www.rpp.com.pe. 
Las empresas que se dedican a examinar países son muchas, pero muy pocas suscitan la confianza y la credibilidad de los inversionistas. Entre ellas tenemos a Standard \& Poor's, Moody's Investors Service, Duff \& Phelps y Fitch IBCA. Estas corporaciones influyen en las decisiones que tomarán los inversionistas para determinar cuántos miles de millones de dólares invertirán en los países; si suben la calificación de un país animarán a los inversionistas a poner su dinero, pero si bajan la calificación los inversionistas podrían retirar su dinero o podrían perderlo. Es por ello que estas empresas son tan importantes; razón de sobra para que los gobiernos realicen intensos cabildeos con ellas.

Los señores calificadores se equivocan de vez en cuando -por supuesto-y quedan como asesores incompetentes. Justamente eso es lo que le pasó a Moody's en 1994 con México: subió su calificación en febrero, animó capitales a venir, llegó la crisis y su prestigio quedó severamente lastimado.

\section{LA OPINIÓN DEL MINISTERIO DE ECONOMÍA Y FINANZAS DEL PERÚ}

Autoridades del gobierno y dirigentes empresariales se congratularon con el grado de inversión otorgado al Perú por la empresa calificadora de riesgo Fitch Ratings. El más optimista fue el entonces Ministro de Economía, Luis Carranza, quien consideró que nuestro país avanza hacia el primer mundo y tras este reconocimiento será más rico. El ministro destacó que las acciones, propiedades y ahorros tendrán mayor valor; además, dijo que este reconocimiento era importante para el país frente a la turbulencia en los mercados internacionales que ha causado pérdidas bancarias y la caída de varias plazas importantes, y que nos coloca entre las primeras economías estables del mundo, lo que podemos considerar como la mejor noticia de los últimos quince años.

La economista Cecilia Blume sostuvo que el grado de inversión tendrá implicancia directa en todos los peruanos y en el crecimiento del país. Permitirá, según dijo, que los recursos nacionales puedan en mayor parte enfocarse en gasto social con el objetivo de combatir la pobreza, además de contribuir a atraer inversión extranjera. No obstante, Blume advirtió que se debe mantener el grado de inversión y no perderlo como consecuencia de un irresponsable manejo económico o político.

\section{LA OPINIÓN DE LOS BANCOS}

El Perú está en mejor posición que la mediana de países con grado de inversión. El nivel de ahorro interno es significativo para hacer frente a contextos internos negativos como fuga de capitales o deterioro en los precios de los metales. Por lo tanto, la vulnerabilidad del Perú frente a shocks externos se reduce, señala Scotiabank en su Reporte Especial ${ }^{2}$.

"Esta buena performance de la economía peruana -en un momento de crisis financiera internacional- difícilmente habría sido posible en el pasado y esto es lo que más ha llamado la atención de los mercados financieros internacionales, en particular de Fitch que le ha otorgado el grado de inversión", agrega el estudio.

Con esto -según Scotiabank- el Perú dejará de ser comparado con países con mayor riesgo como Colombia o Panamá y empezará a serlo frente a economías que generan una mayor confianza sobre los mercados financieros, como la chilena o la mexicana. "La calificación de Fitch es aún más destacable pues se da en un entorno financiero internacional muy turbulento e incierto. Por lo general, estos contextos se asocian típicamente con mayores percepciones de riesgo generalizado". Indica que es muy oportuno también en la medida que este año nuestro país estará en la escena internacional debido a las diversas cumbres internacionales que se celebrarán en el Perú, lo que podría acrecentar el ingreso de inversionistas de largo plazo hacia la economía peruana ${ }^{3}$.

¿Qué cambió o mejoró para que Fitch le otorgue a Perú el grado de inversión? Scotiabank comenta que Perú ha mejorado significativamente sus indicadores macroeconómicos y, en particular, ha tenido una notable mejora en la capacidad del país de atender la deuda pública. Precisa que en el 2007, el Perú pasó de ser un país deudor neto a uno acreedor neto; por lo tanto, los mercados financieros tienen la percepción que tenemos capacidad e interés de pagar la deuda. Asimismo

2 Ver www.scotiabank.com.pe

3 Recordemos que el documento fue elaborado en el 2008, año en que el Perú fue sede de las cumbres mundiales de APEC y ALC-UE. 
detalla que el Perú ha acelerado su crecimiento, pasando de $7 \%$ a $9 \%$, además que, en los últimos doce meses, las Reservas Internacionales Netas (RIN) han pasado de US\$ 18,135 millones a US\$ 32,306 millones (78\%) -con esto, las RIN superan en poco más de US\$ 10,000 millones el stock de deuda pública externa. Por otro lado, en los últimos meses y en un entorno financiero internacional particularmente convulsionado, nuestro país ha realizado diversas operaciones de pre-pago de deuda (Club de París, bonos Brady) y operaciones de Swap de monedas.

\begin{abstract}
"Esto ha contribuido a que los indicadores de solvencia fiscal y externa de Perú se aproximen (y en algunos casos superen) a los mostrados por los países grado de inversión. La capacidad de pago de la deuda de Perú en términos de sus ingresos es similar a la de los países grado de inversión, es decir que el crecimiento de los ingresos del país (PBI, recaudación tributaria) asegura una capacidad de pago similar a la de países con un importante nivel de confianza en los mercados financieros internacionales".
\end{abstract}

Sin embargo, Scotiabank aclara que el otorgamiento a Perú del grado de inversión no asegura el éxito económico per se.

"Varios países con grado de inversión han registrado contracciones económicas al igual que el resto de países o registran problemas estructurales como institucionalidad frágil, deficiente infraestructura, altos niveles de pobreza y/o desigualdad, etc. Incluso, se han dado casos en los que países que no fueron bien manejados a nivel de su macroeconomía se les redujo la calificación".

\section{LA OPINIÓN DE LOS EMPRESARIOS}

En medio de los problemas financieros que aquejan a varios países del mundo, la obtención del grado de inversión le permitirá al Perú distinguirse en el contexto económico internacional, afirmó Jaime Cáceres Sayán, presidente de la Confederación Nacional de Instituciones Empresariales Privadas (CONFIEP), todo esto debido a la fuerte mejora de los indicadores de solvencia externa y fiscal de Perú, que contrarrestan cualquier posibilidad de incumplimiento de pago de sus deudas.

Para el representante gremial, la nueva calificación crediticia otorgada por Fitch a la economía local redundará en beneficio de toda la sociedad peruana, porque permitirá que vengan los grandes inversionistas que no consideraban al Perú porque no tenían la confianza suficiente o porque las regulaciones les imponían restricciones, lo que generará más trabajo en mediano y largo plazo.

"Por donde se mire, ésta es una buena noticia" opinó, para agregar que es un mensaje para los opositores políticos que sugieren cambiar el modelo económico vigente. Por el contrario -comentó- esta buena nota confirma que hay que persistir en este modelo económico y seguir mostrando al mundo que el Perú es capaz de ser un país confiable, seguro, que respeta las inversiones, etc.

Sin embargo, explicó que no todo es color de rosa

"porque ahora corresponde persistir en el mantenimiento de las variables macroeconómicas, y que esto sea una motivación para mejorar la competitividad, mejorar la infraestructura, etc. Cada uno, empresario y trabajador, tiene tareas que cumplir".

Asimismo comentó que la mejora en la calificación de Perú se concretó en un momento preciso, tomando en cuenta que el Perú fue anfitrión de las cumbres América Latina, Caribe y la Unión Europea (ALC-UE) -desarrollada en mayo-y del Foro de Cooperación Económica Asia - Pacífico (APEC), realizada en noviembre pasado.

El presidente de la Cámara de Comercio de Lima (CCL), Samuel Gleiser, sostuvo que la decisión de Fitch Ratings "representa un espaldarazo a la economía peruana”. Según Gleiser, "esto va a generar una mayor posibilidad de inversiones de fuente extranjera. También contribuirá a reducir efectivamente los costos financieros".

El presidente de la Sociedad Nacional de Industrias ( $\mathrm{SNI}$ ), Eduardo Farah, afirmó que el grado de inversión no solo beneficiará a la macroeconomía sino que será un motor importante para el desarrollo de la microeconomía, ya que impulsará la creación de nuevos puestos de trabajo.

Alan Kessel del Río, presidente de la Cámara Nacional de Comercio, Producción y Servicios (PERUCÁMARAS), gremio empresarial que agrupa a 56 cámaras de comercio del interior del país, expresó que

“... el grado de inversión otorgado al Perú por la calificadora de riesgo Fitch Ratings es una noticia realmente alentadora, pero esta 
calificación no es permanente, razón por la cual el gobierno debe trabajar para mantener, en líneas generales, la estabilidad política, económica y jurídica en el país". "Se ha señalado que estamos en camino hacia el primer mundo; el recorrido será bastante largo pero la calificación obtenida por el Perú es importante considerando que el contexto internacional se muestra turbulento".

Kessel del Río explicó que el empresariado del interior del país recibió con mucha expectativa la noticia, pues esta calificación de la deuda peruana de largo plazo implicará nuevas inversiones, las mismas que deberían orientarse a cubrir el fuerte déficit en infraestructura existente, y para ello el gobierno debe propiciar las condiciones necesarias para que éstas se concreten. Precisó que sólo en infraestructura portuaria, el déficit asciende a US\$ 1,000 millones, y que puertos como Paita requieren, en el corto plazo, de una inversión de US\$ 115 millones; Pisco, US\$ 53.3 millones; Ilo, US\$ 19 millones; Iquitos, US\$ 18 millones; y, Pucallpa, US\$ 22 millones. El líder empresarial puso de relieve la necesidad de que estas inversiones se realicen de forma descentralizada para superar los índices de pobreza que en algunas regiones del sur del país continúan siendo altos.

"El grado de inversión es un respaldo al manejo en general de la economía, pero también debe traducirse en una mejora en la calidad de vida de los más necesitados y olvidados del interior del país. Nos preocupa sobremanera el déficit en infraestructura, motivo por el cual hay que trabajar fuertemente en revertirlo, lo cual contribuirá a generar más empleo y a reducir la pobreza”, puntualizó Kessel del Río.

Pero quien reventó cohetes por todo lo alto fue el Presidente del Consejo de Empresarios de América Latina (CEAL): "El grado de inversión es un gran logro del Perú que le permitirá un mayor crecimiento y atraer más inversiones", destacó Fernando Illanes en Palacio de Gobierno, luego de una reunión de 90 minutos con el jefe de Estado, Alan García Pérez. "Es un gran logro (el grado de inversión) que le va a permitir mejorar la situación del país, atraer la inversión y obviamente mejorar aún más en su crecimiento económico", dijo el máximo directivo del CEAL, de nacionalidad boliviana, quien fue recibido por el mandatario conjuntamente con otros 15 miembros del directorio de dicha institución que reúne a 300 empresarios de Argentina, Brasil, Bolivia, Colombia, Chile, Ecuador, México, Paraguay, Perú, Uruguay y Venezuela. Esta organización constituida por los más importantes empresarios privados de América Latina, fue creada con el objetivo principal de estimular la participación de sus miembros en las corrientes de intercambio y cooperación entre los países latinoamericanos.

\section{QUÉ DICEN LOS POLÍTICOS}

El ex ministro de Economía del régimen anterior, Pedro Pablo Kuczinsky, consideró que si bien el grado de inversión otorgado por la empresa calificadora de riesgo Fitch Ratings es un gran espaldarazo para el Perú, aún faltaría obtener el respaldo de Standard \& Poor's, una calificadora de mayor dimensión que Fitch, para que los grandes fondos norteamericanos intervengan en el Perú. Kuczinsky también señaló que si bien el Perú ha registrado siete años de crecimiento continuo sigue siendo un país pobre, donde aproximadamente el $40 \%$ de peruanos vive con menos de US\$ 2 por día y hay una carencia de infraestructura. "Esto es simplemente un peldaño en una larga escalera para modernizar el Perú”, expresó.

\section{DEFINIENDO LA CALIFICACIÓN DE RIESGO}

Pero analicemos técnicamente el concepto de grado de inversión. Para ello nos basaremos en un documento emitido en noviembre del 2007 por el Banco Central de Reserva del Perú (BCRP) ${ }^{4}$.

"En términos generales el rating o calificación de riesgo es la opinión actual de las agencias sobre el riesgo de un emisor de deuda respecto al cumplimiento de una determinada obligación o programa financiero. En particular, la calificación de riesgo o rating soberano es la opinión sobre la habilidad y la disposición de un gobierno soberano de pagar puntualmente todas sus obligaciones financieras, es decir, es una estimación de la probabilidad futura de que un gobierno caiga en cesación de pagos (default)".

"Es importante señalar que el rating soberano se refiere sólo a la capacidad del gobierno central de honrar sus deudas con el sector privado. Los ratings son, entonces, una esti-

4 Choy Chong, Gladis (2007). 
CUADRO N. ${ }^{\circ} 1$

CATEGORÍAS DE RATING DE DEUDA A LARGO PLAZO

\begin{tabular}{|c|c|c|c|}
\hline RIESGO DE CRÉDITO & MOODY'S & S\&P y Fitch & POSICIÓN \\
\hline \multicolumn{4}{|l|}{ Grado de inversión } \\
\hline $\begin{array}{l}\text { Riesgo mínimo de crédito o capacidad de pago extremada- } \\
\text { mente fuerte. }\end{array}$ & Aaa & AAA & 1 \\
\hline Muy bajo riesgo de crédito o muy fuerte capacidad de pago. & Aa1; Aa2; Aa3 & $\mathrm{AA}+; \mathrm{AA} ; \mathrm{AA}-$ & $2-4$ \\
\hline Bajo riesgo de crédito o fuerte capacidad de pago. & $\mathrm{A} 1 ; \mathrm{A} 2 ; \mathrm{A} 3$ & $A+; A ; A-$ & $5-7$ \\
\hline Moderado riesgo de crédito o adecuada capacidad de pago. & Baa1; Baa2; Baa3 & $\mathrm{BBB}+; \mathrm{BBB} ; \mathrm{BBB}-$ & $8-10$ \\
\hline \multicolumn{4}{|l|}{ Grado especulativo } \\
\hline $\begin{array}{l}\text { Sustancial riesgo de crédito: está expuesto a factores adversos } \\
\text { que pueden llevarlo a una inadecuada capacidad de pago. }\end{array}$ & $\mathrm{Ba} 1 ; \mathrm{Ba} 2 ; \mathrm{Ba} 3$ & $\mathrm{BB}+; \mathrm{BB} ; \mathrm{BB}-$ & $11-13$ \\
\hline $\begin{array}{l}\text { Alto riesgo de crédito: está expuesto a factores adversos que } \\
\text { lo llevarán a un deterioro de su capacidad de pago. }\end{array}$ & B1; B2; B3 & $\mathrm{B}+; \mathrm{B} ; \mathrm{B}-$ & $14-16$ \\
\hline $\begin{array}{l}\text { Muy alto riesgo crediticio: es actualmente vulnerable y depende } \\
\text { de un entorno favorable para cumplir sus compromisos. }\end{array}$ & Caa1; Caa2; Caa3 & $\begin{array}{l}\mathrm{CCC}+; \quad \mathrm{C} \mathrm{CC} ; \\
\mathrm{CCC}-\end{array}$ & $17-19$ \\
\hline Cese de pagos inminente: es altamente vulnerable. & Ca. & $\mathrm{CC} ;-\mathrm{C}$ & $20-21$ \\
\hline Cese de pagos. & C & D & 22 \\
\hline
\end{tabular}

Fuente: Moody's, S\&P y Fitch.

mación de riesgo soberano pero no referido a la deuda bilateral con países miembros del Club de París, ni a la contraída con organismos multilaterales como del FMI, BID o BIRF, ni a la contraída con gobiernos subnacionales $o$ empresas estatales. Sin embargo, en muchos procesos de default, las dificultades de pago pueden alcanzar a todas las fuentes acreedoras y entidades emisoras".

"Dependiendo de la agencia, la calificación puede incorporar algunas expectativas de recuperación del principal. Los ratings de Moody's tienen indicadores de pérdida esperada; Fitch evalúa sólo la probabilidad de una cesación de pagos antes de que ocurra, mientras que Standard \& Poor's busca simplemente reflejar la probabilidad de default sin referirse a su magnitud, términos de una posible renegociación o la magnitud de una eventual recuperación del principal"5.

\section{NIVELES Y SIGNIFICADO DE LAS CALIFICACIONES}

En términos generales se definen dos grandes categorías: grado de inversión y grado de es- peculación. Cada categoría, a su vez, está dividida en subcategorías y niveles según la naturaleza del instrumento de deuda calificada. Para diferenciar estas categorías Moody's utiliza números $(1,2$, 3 ), mientras que Standard \& Poor's y Fitch tienen un ranking basado en símbolos (+,-).

\section{EL CONTEXTO INTERNACIONAL}

La siguiente pregunta es pertinente: ¿qué hace Chile?, porque es el principal productor y exportador de cobre en el mundo, con $35.2 \%$ de participación en el mercado y tiene las mayores reservas del planeta. Según datos de la gubernamental Comisión Chilena del Cobre (COCHILCO), en el 2006 el país produjo 5,360.80 tn de concentrado de cobre, casi 5 veces más que su cercano competidor, Estados Unidos de Norte América, que totalizó 1,226 tn. Les siguió Perú, con 1,048.9 unidades $^{6}$. El Estado Chileno sólo controla el 30\% de esa producción a través de la Corporación Nacional del Cobre (CODELCO); el 70\% restante está en manos privadas.

Entre las propuestas planteadas por sectores políticos y sociales para aprovechar el boom están

5 Otaviano, Canuto; Fonseca P. Dos Santos, Pablo y De Sá Porto, Pablo. (2004).

6 Estrada, Daniela. "Desafíos 2008: Chile ante el inquietante boom del Cobre”. En: http://ipsnoticias.net/nota. asp?idnews $=87070$. 
mejorar la educación, invertir en bienes de capital público (principalmente para la salud), apoyar a las pequeñas y medianas empresas, y destinar recursos a las regiones. Allí, como en el Perú, también tienen sus contradicciones, según opina Orlando Caputo, director del Centro de Estudios de Transnacionalización, Economía y Sociedad ${ }^{7}$ :

"En Chile no hay una estrategia de desarrollo del Estado. Esa palabra está prohibida. Todo se lo deja al mercado. Sólo se habla de mejorar los factores productivos, como el capital humano y de generar las condiciones para que haya mayor productividad".

Como puede verse, en todas partes se cuecen habas.

\section{CONCLUSIONES}

1. Alcanzar esta clasificación que analizamos es favorable para las empresas que invierten en el Perú, dada la reducción de la prima de riesgo que los países en desarrollo deben pagar por su mayor probabilidad de impago y mal historial crediticio, lo que generará un mejor ambiente de negocios para los inversionistas extranjeros dentro del país, facilitando establecer proyectos de más largo plazo y permitiéndoles generar perspectivas de inversión de manera más acertada. El ingreso per cápita mejorará para determinados sectores porque la distribución de la riqueza aún es un problema pendiente en las políticas del país. La pobreza en Huancavelica, que alcanza al $84 \%$ de su población, o la mínima inversión privada en Puno, por sólo mencionar dos regiones, generan una frustración enorme en el país.

2. La única forma de generar más empleo, reducir la pobreza, elevar el nivel de vida de los peruanos e incrementar su esperanza de vida es que el gobierno de turno maneje sabiamente las futuras inversiones, orientándolas hacia las regiones con elevados índices de pobreza, además de reducir el déficit en infraestructura vial y portuaria. Esto hace imperativo fortalecer a PROINVERSIÓN y no eliminarlo, y atomizar el control de las inversiones nacionales. Ya no basta con batir palmas por el crecimiento del PBI y el repunte económico que están impulsados por el emprendimiento empresarial privado y no por la sagacidad del régimen para incentivarlo.

3. Es necesario planificar el cumplimiento de las leyes laborales y tributarias, y exigir que se cumplan. Además es importante lograr que el reparto de los beneficios sea equitativo para todas las fuerzas vivas que hacen que la economía del país funcione; única manera de practicar la responsabilidad social empresarial en un marco de buen gobierno corporativo.

\section{BIBLIOGRAFÍA}

Choy Chong, Gladys. (2007). Perú: Grado de Inversión, un Reto de Corto Plazo. Serie de Documentos de Trabajo. Lima, Banco Central de Reserva del Perú, enero 2007.

Otaviano, Canuto; Fonseca P. Dos Santos, Pablo y De Sá Porto, Pablo. (2004). Macroeconomics and Sovereign Risk Ratings. Washington, january 2004.

\section{INTERNET}

www.rpp.com.pe.

www.scotiabank.com.pe

Estrada, Daniela. "Desafíos 2008: Chile ante el inquietante boom del Cobre". En: http:// ipsnoticias.net/nota.asp?idnews $=87070$.

7 Citado en el artículo anteriormente mencionado. 